\title{
Cadre d'indicateurs des maladies chroniques et des blessures Statistiques rapides, édition automne 2014
}

\section{Division de la surveillance et de l'épidémiologie, Centre de prévention des maladies chroniques, Agence de la santé publique du Canada}

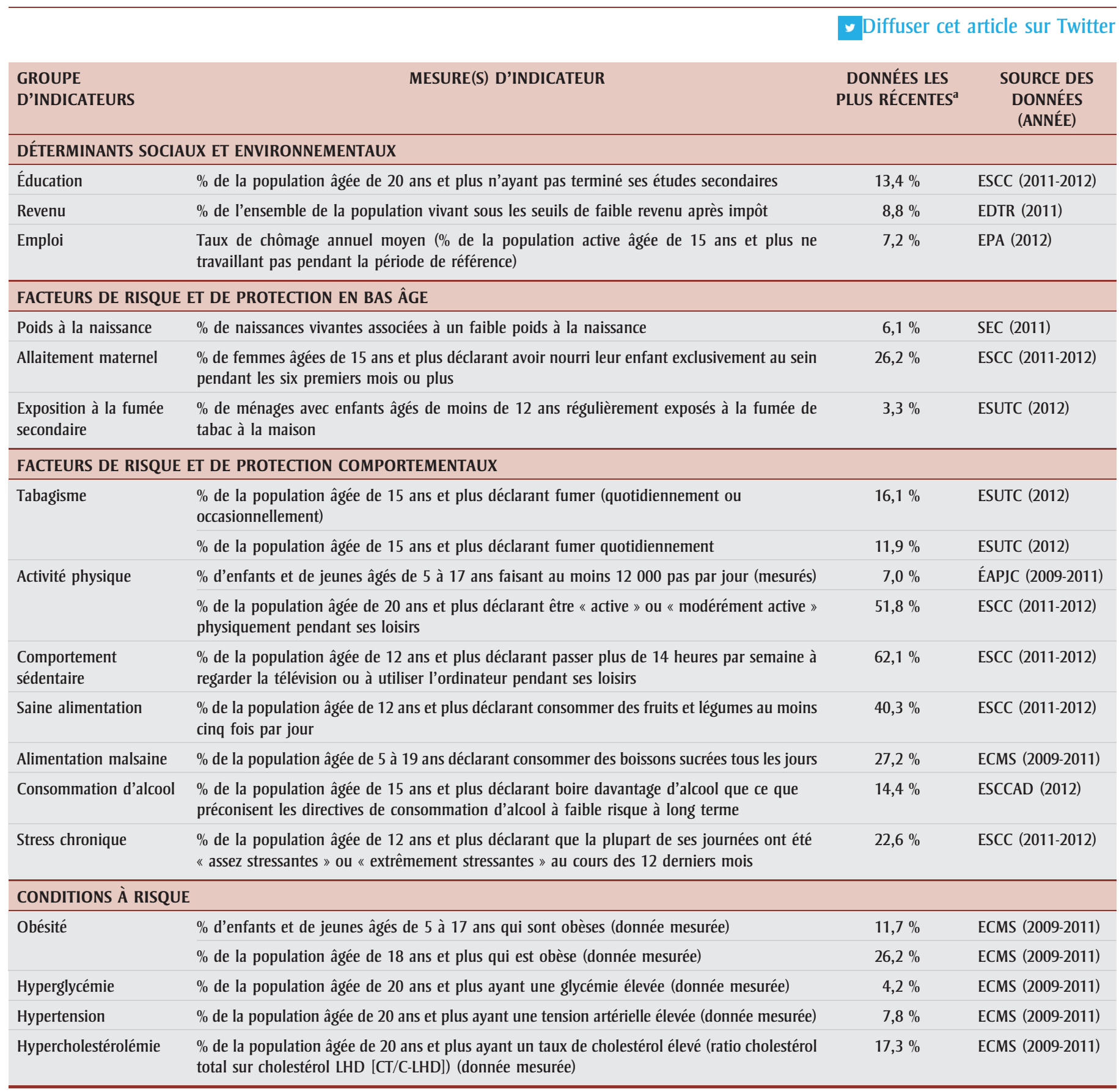




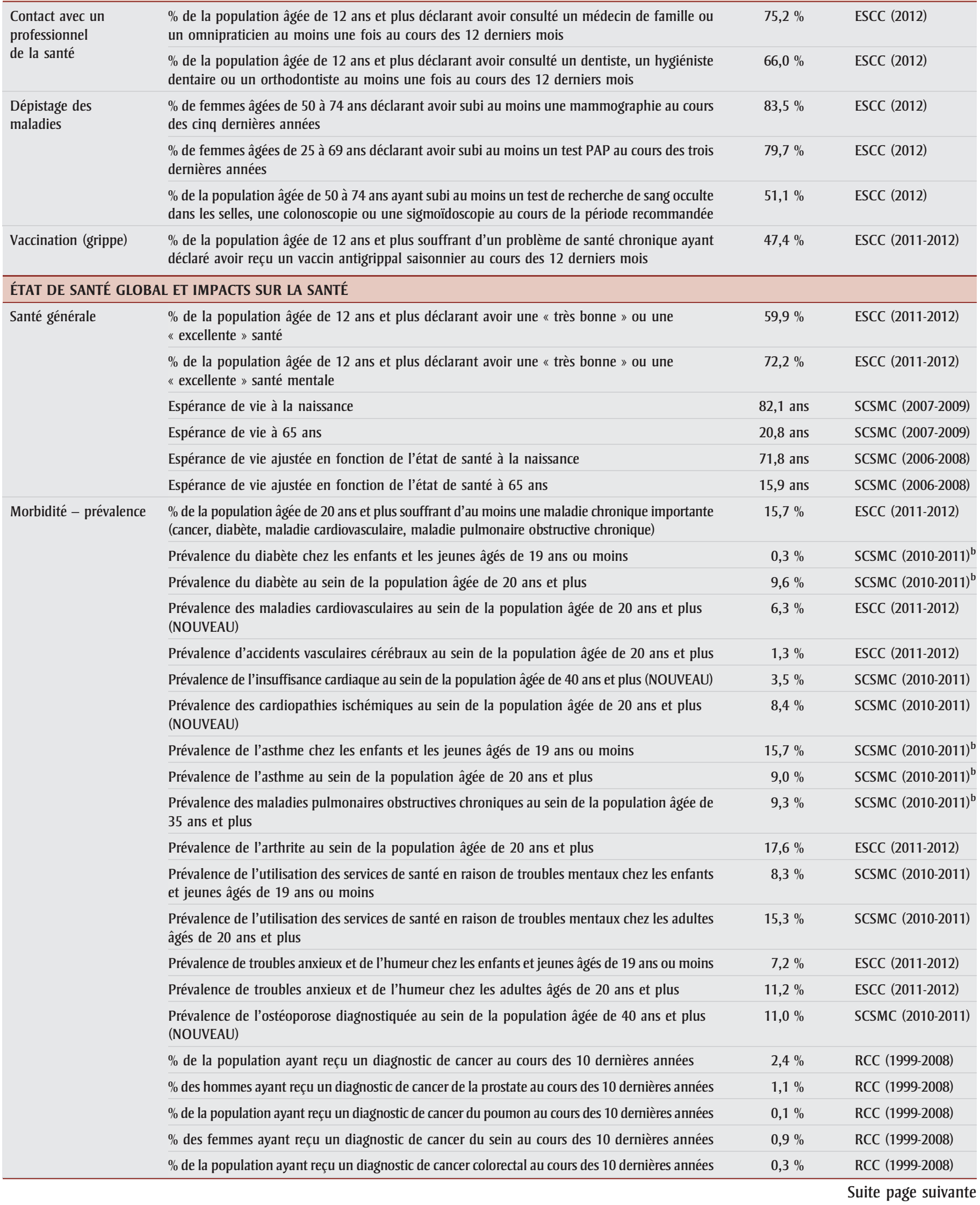




\section{ÉTAT DE SANTÉ GLOBAL ET IMPACTS SUR LA SANTÉ}

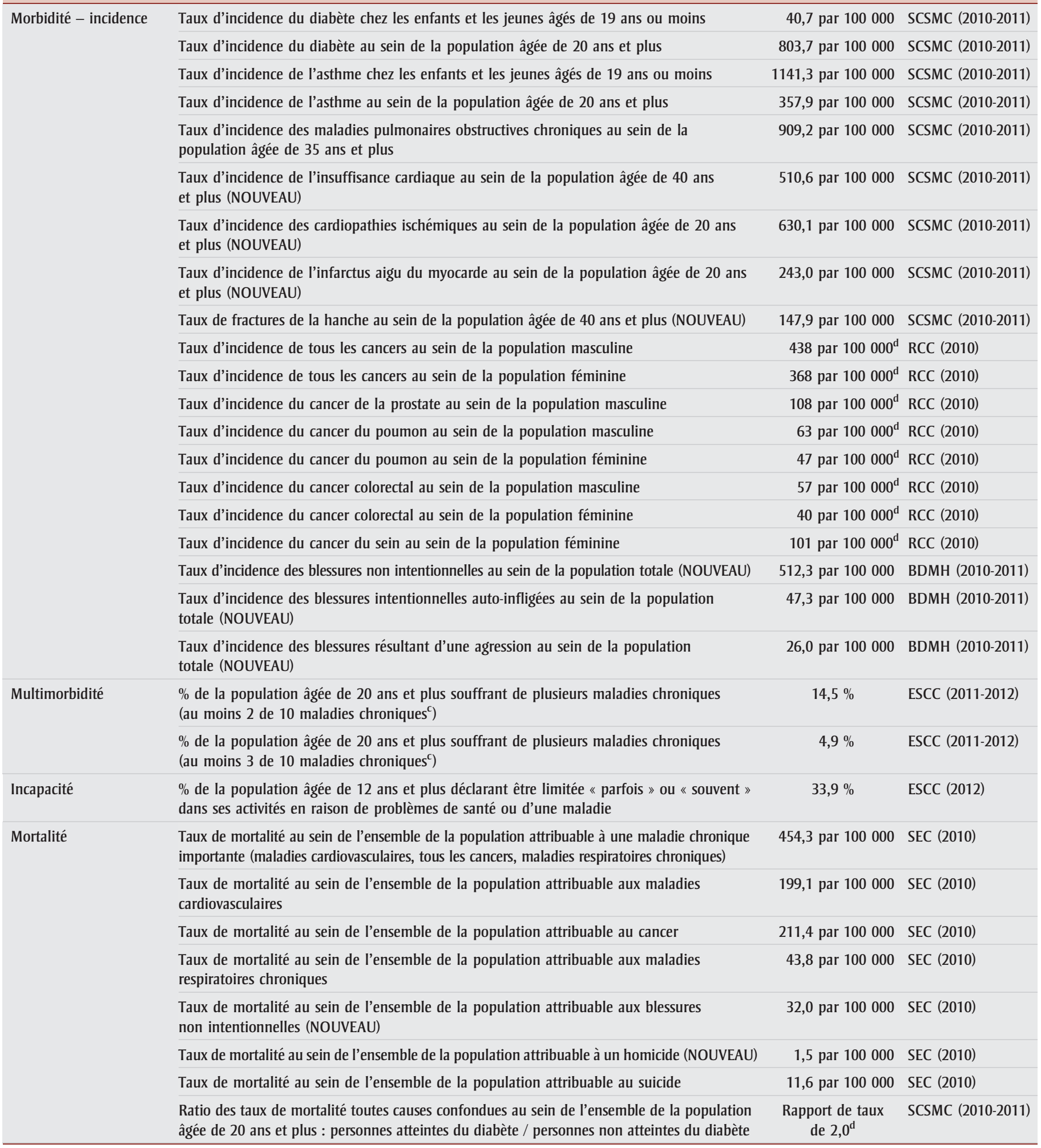




\section{ÉTAT DE SANTÉ GLOBAL ET IMPACTS SUR LA SANTÉ}

\begin{tabular}{|c|c|c|c|}
\hline \multirow[t]{6}{*}{ Mortalité prématurée } & Années potentielles de vie perdues en raison du cancer & 1480,6 par 100000 & SEC (2010) \\
\hline & Années potentielles de vie perdues en raison des maladies cardiovasculaires & 733,1 par 100000 & SEC (2010) \\
\hline & Années potentielles de vie perdues en raison des maladies respiratoires chroniques & 118,8 par 100000 & SEC (2010) \\
\hline & $\begin{array}{l}\text { Probabilité de mourir (\%) entre } 30 \text { et } 69 \text { ans de maladies chroniques importantes } \\
\text { (maladies cardiovasculaires, cancer, maladies respiratoires chroniques, diabète) }\end{array}$ & $11,0 \%$ & SEC (2010) \\
\hline & Probabilité de mourir (\%) entre 30 et 69 ans d'une maladie respiratoire chronique & $0,7 \%$ & SEC (2010) \\
\hline & Probabilité de mourir (\%) entre 30 et 69 ans du diabète & $0,5 \%$ & SEC (2010) \\
\hline
\end{tabular}

Abréviations : BDMH, Base de données sur la morbidité hospitalière; cholestérol LHD, cholestérol à lipoprotéines de haute densité; CT, cholestérol total; ÉAPJC, Étude sur l'activité physique des jeunes au Canada; ECMS, Enquête canadienne sur les mesures de la santé; EDTR, Enquête sur la dynamique du travail et du revenu; EPA, Enquête sur la population active; ESCC, Enquête sur la santé dans les collectivités canadiennes; ESCCAD, Enquête de surveillance canadienne de la consommation d'alcool et de drogues; ESUTC, Enquête de surveillance de l'usage du tabac au Canada; RCC, Registre canadien du cancer; SCSMC, Système canadien de surveillance des maladies chroniques; SEC, Statistique de l'état civil.

Remarque : Les taux calculés par le SCSMC n’incluent pas les données de l’Alberta. Les taux calculés par le SEC n'incluent pas les données du Québec.

a Tous les taux présentés sont bruts sauf indication contraire.

b Des données de l'ESCC de 2011-2012 existent pour cet indicateur et peuvent être ventilées selon des variables démographiques et sociales.

c Multimorbidité : Les maladies chroniques incluses sont les maladies du cœur, les accidents vasculaires cérébraux, les cancers, l'asthme, la maladie pulmonaire obstructive chronique, le diabète, l'arthrite, la maladie d'Alzheimer et autres démences, les troubles anxieux et de l'humeur (dépression).

${ }^{\text {d }}$ Les taux ont été normalisés en fonction de l'âge de la population au Canada en 1991.

Citation suggérée : Centre de prévention des maladies chroniques, Agence de la santé publique du Canada (2014). Cadre d’indicateurs des maladies chroniques, édition automne 2014.

Si vous avez des questions ou des commentaires, veuillez communiquer avec nous à l'adresse suivante : Chronic.Publications.Chronique@phac-aspc.gc.ca

Utilisez l'outil en ligne du cadre d'indicateurs des maladies chroniques afin de voir la répartition de données additionnelles : http://infobase.phac-aspc.gc.ca/cdif/?l = fra 